\title{
Outcome of early coronary intervention for acute ST elevation myocardial infarction in a tertiary care cardiac centre in Sri Lanka
}

\author{
M B F Rahuman ${ }^{1}$, J B Jayawardana ${ }^{1}$, G R Francis ${ }^{1}$, M Niraj ${ }^{1}$, A H T W Kumara ${ }^{1}$, U A D Wijesinghe ${ }^{1}$, \\ R Haniffa ${ }^{2,3,4}$, R Ariyapperuma ${ }^{1}$, C Anuruddha ${ }^{1}$, A P De Silva ${ }^{2,5}$ \\ (Index words: percutaneous coronary intervention, myocardial infarction, Sri Lanka, outcome)
}

\begin{abstract}
Objectives To describe the outcomes of early percutaneous coronary intervention $(\mathrm{PCl})$ for the treatment of acute ST elevation myocardial infarction (STEMI) in a tertiary care cardiac centre in Colombo, Sri Lanka.

Methods Medical records of 139 consecutive patients presenting to Cardiology Unit 5, National Hospital of Sri Lanka from March 2013 to June 2014 with acute STEMI, and treated with early $\mathrm{PCl}$ as a mode of reperfusion were reviewed. These patients were then followed up for 6 months to determine survival, targetvessel revascularization, in-stent thrombosis and other major adverse cardiac events (MACE).

Results Of 139 patients, 116 (83.5\%) were male. Mean age was $52.3 \pm$ SD11.1 years. Eighty eight (63.3\%) patients underwent primary $\mathrm{PCl}$ and 51 (36.7\%) underwent rescue $\mathrm{PCl}$. There were six deaths (4.3\%). One occurred on-table and three occurred after discharge. Four patients who died had cardiogenic shock. Mean door-to-balloon (DTB) time was 147 minutes for the primary $\mathrm{PCI}$ patients who were transferred from ETU. At six months, of 106 patients who attended follow up, two had been re-hospitalised for heart failure but none underwent coronary artery bypass grafting (CABG).
\end{abstract}

Conclusions This report from the national tertiary care cardiology referral centre in Sri Lanka, found that the study population was relatively younger, similar to other Asian countries. There was high rate of initial success (98.6\%) and good short-term survival (95.7\%), particularly in the subset presenting without cardiogenic shock (98.4\%) despite the long DTB time. Loss to follow up at 6 months in this centre was $23.7 \%$ (33 patients).

Ceylon Medical Journal 2016; 61: 26-31

\section{Introduction}

Cardiovascular diseases, including myocardial infarction (MI) and heart failure, are the leading causes of death in developed as well as in low-and middle-income countries (LMICs) [1]. Myocardial infarction is the leading cause of death in Sri Lanka and risk factors such as diabetes, hypertension, smoking and obesity are becoming commoner [2]. Although considered primarily a disease of the middle and old age, recent trends in Asia suggest increasing prevalence of MI in the young [3]. When treated promptly the outcome of younger patients with ST elevation myocardial infarction (STEMI) is better than in older patients [4].

The aim of acute treatment of STEMI is restoration of myocardial perfusion by recanalisation of the occluded vessel. Early reperfusion is associated with a better outcome [5,6]. Many studies have shown that early coronary intervention reduces mortality and morbidity compared to thrombolysis alone [7-9].

Fibrinolytic therapy has been an important means of establishing reperfusion for decades. However, there are many limitations to the use of thrombolytic therapy due to high rates of recurrent ischaemia and re-occlusion [9]. Even with best thrombolytic agents such as tenecteplase and alteplase, $20 \%$ of occluded arteries are not reperfused leading to persistent ischaemia. However streptokinase, which is currently the only widely available fibrinolytic agent in Sri Lanka has a much lower rate of re-perfusion (40-50\%) leading to higher rates of persistent myocardial damage and heart failure which contribute to increased morbidity and mortality [10]. Therefore, use of percutaneous coronary intervention (PCI) is the preferred re-perfusion strategy, either as primary PCI or PCI secondary to thrombolysis, depending on resource availability [7]. Evidence from high income countries

DOI: http://doi.org/10.4038/cmj.v61i1.8258

${ }^{1}$ Institute of Cardiology, National Hospital of Sri Lanka, ${ }^{2}$ National Intensive Care Surveillance, ${ }^{3}$ Mahidol Oxford Tropical Medicine Research Unit (MORU), ${ }^{4}$ Faculty of Medicine, University of Colombo, ${ }^{5}$ Intensive Care National Audit and Research Centre, Sri Lanka.

Correspondence: MBFR, e-mail: <faslurcard@yahoo.com>. Received 30 March 2015 and revised version accepted 15 November 2015.

This is an open-access article distributed under the terms of the Creative Commons Attribution License, which permits unrestricted use, distribution, and reproduction in any medium, provided the original author and source are credited. 
(HICs) suggests that primary PCI should be the preferred method of treatment for STEMI, provided that the procedure could be performed within 90 minutes of initial patient contact, subject to the availability of skilled interventional cardiologists, equipped centres and surgical backup [11]. Although PCI has been available for many years in Sri Lanka there is a paucity of data related to patient outcome [12].

The aim of this study was to describe the outcome of early PCI for acute STEMI in a tertiary care cardiac centre in Colombo, Sri Lanka.

\section{Methods}

This was a cohort study conducted at Cardiology Unit, National Hospital of Sri Lanka (NHSL). Consecutive patients presenting with an acute STEMI who underwent primary or rescue PCI during the study period from March 2013 to June 2014 were recruited at the time of PCI and followed up for a period of six months. Those who received prior thrombolysis at the first contact point for the current event, underwent rescue PCI, while those who did not receive prior thrombolysis at the first contact point underwent primary PCI. STEMI was diagnosed when there was chest pain and ST elevation in two consecutive leads or new onset LBB (left bundle branch block) in the electrocardiogram (ECG) [13]. ST elevation in ECG was diagnosed when there was ST elevation at the J point in at least two contiguous leads of $\geq 2 \mathrm{~mm}(0.2 \mathrm{mV})$ in men or $1.5 \mathrm{~mm}(0.15 \mathrm{mV})$ in women in leads V2-V3 or $1 \mathrm{~mm}(0.1 \mathrm{mV})$ in other contiguous chest leads or the limb leads [14]. Rescue PCI was carried out after failed thrombolysis in patients who had poor $(<50 \%)$ ST resolution in 90 minutes, persistent chest pain or cardiogenic shock.

Data were extracted prospectively from patient notes using data sheets. Follow up data were extracted from clinic records at two weeks after leaving hospital and monthly thereafter. Patients who did not attend follow up were contacted over the telephone. Socio-demographic information and selected risk factors of heart disease including lifestyle and baseline clinical features were recorded. Lifestyle was categorised as active or sedentary based on the occupation. Those who were skilled, semiskilled and manual workers were categorised as having an active lifestyle and professional, technical and clerical grades were categorised as having a sedentary lifestyle [15].

Persistent ST elevation was defined as less than $50 \%$ resolution of ST elevation when the admission ECG (taken at point of first medical contact) was compared to postprocedure ECG. Depending on the regional ECG changes MI was classified as anterior STEMI, antero-lateral STEMI, inferior STEMI and posterior STEMI. The presence of cardiogenic shock was defined as patients who required inotropic support to maintain a minimum systolic blood pressure of $90 \mathrm{mmHg}$ during or before the PCI. The time taken from development of symptoms to hospital admission (according to patient records) was defined as symptom-to-door time (SDT), and time from admission to re-establishment blood flow in the affected artery (as determined by fluoroscopy) was defined as the door-toballoon time (DBT). PCI procedures for working hours were defined as those carried out on week days from 800 hours to 1600 hours.

The vessel affected and the degree of obstruction were recorded using fluoroscopic angiography by the consultant cardiologist. Concomitant involvement of other vessels (non-culprit vessels with over $70 \%$ plaque disease in the angiogram) was documented.

Ejection fraction was measured on admission, before leaving hospital, at six weeks and at six months by the Cardiologist or the Senior Registrar. Following the PCI, the patients were assessed for clinical improvement of chest pain. A 12 lead ECG was taken following the PCI procedure to assess resolution of ST changes. A coronary angiogram was performed before, during and after the PCI procedure and the flow results were recorded. The final flow was graded using Thrombolysis in Myocardial Infarction (TIMI) grading [16]. TIMI grade 3 coronary flow of the treated vessel and a residual stenosis less than $30 \%$ were taken as a successful PCI.

All patients received $100 \mathrm{U} / \mathrm{kg}$ of unfractionated heparin intravenously, oral aspirin (300 mg), oral clopidogrel (300 mg/ $600 \mathrm{mg}$ loading dose) and atorvastatin 40 mg. Some received GpIIb-IIIa inhibitors during PCI. Postinterventional therapy for all patients included aspirin (150 $\mathrm{mg}$ ), clopidogrel (75 mg) and atorvastatin $40 \mathrm{mg}$. Stenting procedures were performed according to standard techniques. The number and length of stents, and type of bare metal stents (BMS)/ drug eluting stents (DES) implanted, were the operator's discretion.

Main outcomes measured were death, re-infarction, target-vessel revascularisation, in-stent thrombosis and other major adverse cardiac events (MACE) at 6 months follow up. MACE included patients undergoing coronary artery bypass graft after PCI and recurrent hospital admissions. All deaths were considered cardiac unless a clear non-cardiac cause could be identified.

Continuous variables with normal distribution were described using mean and standard deviation while median and inter quartile range were used for those with a skewed distribution. Discrete variables were described using count and percentage. $\mathrm{T}$ test was used for comparison of continuous variables while chi-square test was used to compare discrete variables. Logistic regression models were used for multivariate analysis. Survival analysis was described using univariate Kaplan-Meier statistics with death at six months follow up as the end point. SPSS 17 and STATA 13 were used for statistical analysis. Approval for the study was obtained from the Ethics Review Committee of NHSL. 


\section{Results}

The study sample included data from 139 consecutive patients who underwent either primary or rescue PCI for acute STEMI, between March 2013 and June 2014. Majority (85.2\%) were males. All patients included in the study underwent early coronary interventions as the treatment choice for STEMI. Eighty eight underwent primary PCI and 51 underwent rescue PCI. Hundred and six (76.3\%) patients were followed up for the full duration of six months. Mean age (SD) of the study participants was 52.3 ( \pm 11.2 years). Mean age of those who underwent primary PCI was 53.2 years and rescue PCI was 50.9 years. Table illustrates the demography, characteristics of inhospital patient management, mortality rate and angiographic features of the study population.

For primary PCI mean SDT was 568.2 minutes (95\%
CI 10 - $4320 \mathrm{~min}$ ) and DTB time was 1093.7 minutes (95\% CI 60 - 5760 min). Mean DTB time was 147 minutes for the primary PCI patients who were directly admitted to emergency treatment unit and transferred to PCI unit which reflects the time taken when patients are directly admitted to a hospital where primary PCI facilities are available.

Mean ejection fraction showed a rapid rise soon after the procedure which ranged from $46 \%$ to $50 \%$. After that it remained constant for the first six weeks of follow up. The mean ejection fraction increased slightly up to 51\% during the next five months. Among PCI patients, there was a similar rise soon after the procedure and reached $55 \%$ at six months. However, in the primary PCI patients, although there was a rise in ejection fraction after the procedure, the mean ejection fraction dropped to $48 \%$ after six weeks and remained so at six months.

Table. Characteristics of patients

\begin{tabular}{|c|c|c|c|}
\hline Characteristic & $\begin{array}{c}\text { Primary PCI }(n=88) \\
\text { Number }(\%)\end{array}$ & $\begin{array}{c}\text { Rescue PCI }(n=51) \\
\text { Number }(\%)\end{array}$ & $\begin{array}{l}\text { Total PCI }(n=139) \\
\text { Number }(\%)\end{array}$ \\
\hline Male & $75(85.2)$ & $41(80.4)$ & $116(83.5)$ \\
\hline \multicolumn{4}{|l|}{ Past medical history: } \\
\hline Hypertension & $26(29.6)$ & $8(15.7)$ & $34(24.5)$ \\
\hline Diabetes mellitus & $23(26.1)$ & $17(33.3)$ & $40(28.8)$ \\
\hline Hyperlipidemia & $15(17.1)$ & $7(13.7)$ & $22(15.8)$ \\
\hline Family history of CAD & $16(18.2)$ & $13(25.5)$ & $29(20.9)$ \\
\hline \multicolumn{4}{|l|}{ Admission characteristics: } \\
\hline Cardiogenic shock & $8(9.1)$ & $8(15.7)$ & $16(11.5)$ \\
\hline Left ventricular failure & $2(2.3)$ & $01(2)$ & $3(2.2)$ \\
\hline Ejection fraction (mean) & $46.5 \%$ & $46.2 \%$ & $46.34 \%$ \\
\hline Anterior myocardial infarction & $54(61.4)$ & $36(70.6)$ & $90(64.7)$ \\
\hline Inferior myocardial infarction & $29(32.9)$ & $14(27.5)$ & $43(30.9)$ \\
\hline \multicolumn{4}{|l|}{ Details of patient management } \\
\hline \multicolumn{4}{|l|}{ Culprit vessel: } \\
\hline Left anterior descending & $53(60.2)$ & $38(74.5)$ & $91(65.4)$ \\
\hline Left circumflex & $3(3.4)$ & $2(3.9)$ & $5(3.6)$ \\
\hline Right coronary & $33(37.5)$ & $11(21.6)$ & $44(31.2)$ \\
\hline Multivessel CAD & $38(43.2)$ & $22(43.1)$ & $60(43.2)$ \\
\hline Procedural success & $86(97.2)$ & $51(100)$ & $137(98.6)$ \\
\hline BMS Stent & $29(32.9)$ & $13(25.5)$ & $42(30.4)$ \\
\hline DES Stent & $52(59.1)$ & $36(70.6)$ & $88(63.8)$ \\
\hline MACE & $7(7.9)$ & $0(0.0)$ & $7(5.0)$ \\
\hline \multicolumn{4}{|c|}{ Glycoprotein IIb / IIIa inhibitors use: } \\
\hline Given & $50(56.8)$ & $29(56.9)$ & $79(56.8)$ \\
\hline Not given & $38(43.2)$ & $22(43.1)$ & $60(43.2)$ \\
\hline \multicolumn{4}{|l|}{ Mortality } \\
\hline Within $24 \mathrm{hrs}$ & $3(3.4)$ & $0(0.0)$ & $3(2.2)$ \\
\hline Within 1 month & $5(5.7)$ & $0(0.0)$ & $5(3.6)$ \\
\hline Within 6 months & $6(6.8)$ & $0(0.0)$ & $6(4.3)$ \\
\hline
\end{tabular}

CAD: Coronary Artery Disease, PCI: Percutaneous Coronary Intervention, MACE: Magor adverse cardiac event 
Five patients who had MACE during the follow-up were aged more than 60 years. Of the seven patients who had MACE, only two (28.6\%) had SDT less than three hours and only one patient had a DBT of less than two hours. Multi-vessel disease was responsible for five of those who had MACE. Figure shows the survival data of the study sample.

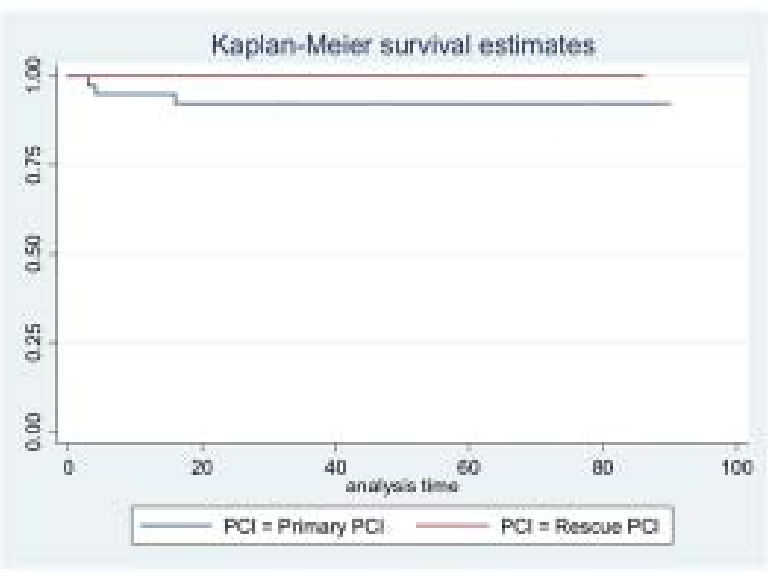

Figure. Kaplan-Meier survival curves for primary and rescue $\mathrm{PCl}$ patients.

\section{Discussion}

This is the first sizeable report on outcomes of primary PCI in acute STEMI from a government funded tertiary care hospital in Sri Lanka. This study describes the results of an unselected "real-world" patient cohort which showed a high success rate $(98.6 \%)$ of the index procedure and an excellent overall in-hospital survival rate (97.8\%), particularly in the absence of cardiogenic shock (almost $98.4 \%)$. Two of 123 patients without cardiogenic shock died (1.6\%) which is similar to international data which shows in-hospital mortality of 5.2\% in the second national registry of myocardial infarction (NRMI2) and 3\% in ASSENT 4 trial [17]. In our study 16 patients had cardiogenic shock and four of them died (25\%) which is similar to available data-32\% in NRMI 2, 46.4\% in SHOCK registry and 59.1\% in American College of CardiologyNational Cardiovascular Data Registry (ACC-NCDR) $[18,19]$.

Several other outcomes of this study compare favourably with those reported from private sector hospitals in Sri Lanka as well as those from HICs [12]. Thus, we suggest that post-PCI outcomes in a tertiary care hospital in a LMIC, at least in the short term, is similar to those from HICs not only for the low-risk (noncardiogenic shock) patients, but even for those presenting with cardiogenic shock. These favourable results were maintained long term, though data is limited by the significant loss to follow up. The rates of initial success as well as TIMI 3 flow were also similar to HICs [13,17, 24].
There are several of findings in our study that merit further discussion. The mean age in our study group was less than 55 years which is lower than those from HIC [25]. Age is a strong prognostic indicator and it is possible that younger patients could have been selected out as a lower-risk cohort for the procedure. However, this lower age at presentation is consistent with other studies on acute MI in the general population of Sri Lanka who have not necessarily undergone primary PCI [26].

Unlike other studies, we did not find a significant association between door-to-balloon time and the outcome of PCI. Only $32.7 \%$ of primary PCI patients had a door-toballoon time of $\leq 120$ minutes. The DBT of our study was significantly higher than those in HICs though our outcomes were similar. Although surprising, this trend has also been observed in the second National Registry of Myocardial Infarction (NRMI-2). This may reflect the inherent difficulties in accurately timing the onset of coronary occlusion (when infarction begins as opposed to the pre-infarct anginal phase) or a survivor cohort effect [18].

There are several implications of our study. Widespread availability of primary PCI, although vigorously promoted, has yet to become a reality, even in the HICs [27]. We have shown that early PCI after STEMI is a viable therapeutic option in the government sector hospitals in Sri Lanka and can be performed with good outcomes despite relatively longer chest pain-topresentation and door-to-balloon times. The key question is whether such programmes can be funded by the state on a widespread scale. This justifies further investigations including disability adjusted life years (DALY), quality of life and cost-effectiveness evaluations. Preservation of the workforce must be a state priority. In the provision of this service on a wider scale, temporary cost-saving measures including the use of cheap bare-metal stents, re-sterilized equipment including guiding catheters, wires and balloons and the involvement of tertiary care hospitals in each province of Sri Lanka to perform primary PCI may be helpful [29].

Our study has several limitations. The sample size was relatively small and only $76.3 \%$ had follow-up data. Although the sample consisted of consecutive patients undergoing primary PCI, they did not represent all patients who presented with acute STEMI. As described in the methods section, many such patients opt for fibrinolysis. Therefore, there may have been a bias towards either affluent (who would buy stents) or patients who were more ill undergoing primary PCI. Furthermore, our data represents a single centre experience where the operators were experienced and the institute was specialized in cardiac services. Whether these results can be generalised to other hospitals in LMICs is uncertain.

In conclusion, the results of this preliminary study raise the possibility that outcomes of PCI after STEMI in LMICs are similar to that in the developed world. 


\section{Acknowledgement}

We acknowledge the support given by the staff of Institute of Cardiology, National Hospital Sri Lanka and the National Intensive Care Surveillance, Ministry of Health.

\section{Conflicts of interests}

There are no conflicts of interest.

\section{References}

1. Gersh BJ, Sliwa K, Mayosi BM, Yusuf S. Novel therapeutic concepts: The epidemic of cardiovascular disease in the developing world: Global implications. Eur Heart J 2010; 31: 642-8.

2. Department of Health. Annual health bulletin 2012 . Colombo; 2014.

3. Reddy KS. Cardiovascular Disease in non-Western Countries. N Engl J Med 2004; 350: 2438-40.

4. Antman EM. ACC/AHA Guidelines for the Management of Patients with ST-Elevation Myocardial InfarctionExecutive Summary: A Report of the American College of Cardiology/American Heart Association Task Force on Practice Guidelines Writing Committee to Revise the 1999. Circulation 2004; 110: 588-636.

5. Boersma E, Maas AC, Deckers JW, Simoons ML. Early thrombolytic treatment in acute myocardial infarction: reappraisal of the golden hour. Lancet 1996; 348: 771-5.

6. De Luca G, Suryapranata H, Ottervanger JP, Antman EM. Time delay to treatment and mortality in primary angioplasty for acute myocardial infarction: every minute of delay counts. Circulation 2004; 109: 1223-5.

7. Grines CL, Browne KF. A comparison of immedicate angioplastry with thrombolytic therapy for acute myocardial infarction. N Engl J Med 1993; 328: 673-9.

8. Pereira H. Long-term benefit of primary angioplasty as compared with thrombolytic therapy for acute myocardial infarction. Rev Port Cardiol 2000; 19: 379-80.

9. Andersen HRHR, Neilsen TT, Rasmussen K, et al. A comparison of coronary angioplasty with fibrinolytic therapy in acute myocardial infarction. N Engl J Med 2003; 349: 733-42.

10. Gurugama P, Matthias AT, Rajkannah J, Kandeepan T. Streptokinase in STEMI: ninety minute ECG for ST resolution; experience in an acute medical unit. $J$ Ceylon Coll Physic 2012; 43: 32-6.

11. O’Connor RE, Brady W, Brooks SC, et al. Part 10: Acute coronary syndromes: 2010 American Heart Association Guidelines for Cardiopulmonary Resuscitation and Emergency Cardiovascular Care. Circulation 2010; 122 (suppl 3): S787-817.

12. Abeysuriya V, Chandrasena LG, Kasturiratne A, et al. Outcome of patients with ST segment elevation myocardial infarction (STEMI) following percutaneous transluminal coronary angioplasty: a retrospective study. Ceylon Med J 2014; 59: 118-23.

13. O'Gara PT, Kushner FG, Ascheim DD, et al. 2013 ACCF/ AHA guideline for the management of st-elevation myocardial infarction: A report of the American college of cardiology foundation/american heart association task force on practice guidelines. J Am Coll Cardiol 2013; 61: e78e140.

14. Sørensen JT, Stengaard C, Sørensen CA, et al. Diagnosis and outcome in a prehospital cohort of patients with bundle branch block and suspected acute myocardial infarction. Eur Heart J Acute Cardiovasc Care 2013; 2: 176-81.

15. Aveline M. Commentary on Cochrane AL (1934). Elie Metschnikoff and his theory of an "instinct de la vie.” Int J Epidemiol 2003; 32: 34-5.

16. Cannon CP. Importance of TIMI 3 flow. Circulation 2001; 104: 624-6.

17. Zalewski J, Bogaerts K, Desmet W, et al. Intraluminal thrombus in facilitated versus primary percutaneous coronary intervention: an angiographic sub study of the ASSENT-4 PCI (Assessment of the Safety and Efficacy of a New Treatment Strategy with Percutaneous Coronary Intervention) trial. J Am Coll Cardiol 2011; 57: 1867-73.

18. Tiefenbrunn a J, Chandra NC, French WJ, et al. Clinical experience with primary percutaneous transluminal coronary angioplasty compared with alteplase (recombinant tissue-type plasminogen activator) in patients with acute myocardial infarction: a report from the Second National Registry of Myocardial. J Am Coll Cardiol 1998; 31: 1240-5.

19. Brindis RG, Fitzgerald S, Anderson HV, et al. The American College of Cardiology-National Cardiovascular Data Registry (TM) (ACC-NCDR(TM)): building a national clinical data repository. J Am Coll Cardiol 2001; 37: 2240-5.

20. Barron H V, Michaels a D, Maynard C, Every NR. Use of angiotensin-converting enzyme inhibitors at discharge in patients with acute myocardial infarction in the United States: data from the National Registry of Myocardial Infarction 2. J Am Coll Cardiol 1998; 32: 360-7.

21. Arshad S, Dhakam S, Awan S. Outcomes in ST elevation Myocardial Infarction; a comparison of a tertiary care center in Pakistan with European centers. J Pak Med Assoc 2011; 61: 1215-9. Available from: http://www.ncbi.nlm.nih.gov/ pubmed/22355970

22. Farman MT, Sial JA, Khan NU. Original Article Outcome of primary percutaneous coronary intervention at public sector tertiary care hospital in Pakistan Data collection: Procedure: Primary PCI of the infarct related artery was. $J$ Pak Med Assoc 2011; 61: 575-81.

23. Ali M, Qadir F, Javed S, et al. Factors affecting outpatient cardiac rehabilitation attendance after acute myocardial infarction and coronary revascularization - a local experience. J Pak Med Assoc 2012; 62 (Phase 1): 347-51.

24. Cannon CP. Evolving management of ST-segment elevation 
myocardial infarction: update on recent data. Am J Cardiol 2006; 98: 10Q - 21Q.

25. Czarnecki A, Prasad TJ, Wang J, et al. Adherence to process of care quality indicators after percutaneous coronary intervention in Ontario, Canada: a retrospective observational cohort study. Open Hear 2015; 2: e200-e208. Available from: http://openheart.bmj.com/cgi/doi/10.1136/ openhrt-2014-000200

26. Wickramatilake CM, Mohideen MR, Pathirana C. Premature coronary artery disease and testosterone in Sri
Lankan men. Ceylon Med J 2014; 59: 17-21.

27. De Belder a. Should primary angioplasty be available for all patients with an ST elevation myocardial infarction? Heart 2005; 91: 1509-11.

28. Lincoff AM, Topol EJ. Illusion of reperfusion. Does anyone achieve optimal reperfusion during acute myocardial infarction? Circulation1993; 88: 1361-74.

29. The reuse of single-use cardiac catheters: safety, economical, ethical and legal issues. Conseild' evaluation des technologies de la sante du Quebec. Can J Cardiol 1994; 10: 413-21. 